\title{
Phase diagram of the $S=\frac{1}{2}$ quantum spin chain with bond alternation
}

\author{
M. Yamanaka, Y. Hatsugait and M. Kohmoto \\ Institute for Solid State Physics, University of Tokyo \\ 7-22-1, Roppongi, Minato-ku, Tokyo 106 Japan
}

We study the ground state properties of the bond alternating $S=1 / 2$ quantum spin chain whose Hamiltonian is

$$
H=\sum_{j}\left(S_{2 j}^{x} S_{2 j+1}^{x}+S_{2 j}^{y} S_{2 j+1}^{y}+\lambda S_{2 j}^{z} S_{2 j+1}^{z}\right)+\beta \sum_{j} \mathbf{S}_{2 j-1} \cdot \mathbf{S}_{2 j} \cdot
$$

When $\beta=0$, the ground state is a collection of local singlets with a finite excitation gap. In the limit of strong ferromagnetic coupling $\beta \rightarrow-\infty$, this is equivalent to the $S=1 X X Z$ Hamiltonian. It has several ground state phases in the $\lambda$ - $\beta$ plane including the gapful Haldane phase. They are characterized by a full breakdown, partial breakdowns and a non-breakdown of the hidden discrete $Z_{2} \times Z_{2}$ symmetry. The ground state phase diagram is obtained by series expansions.

PACS numbers: 75.10.Jm, 75.50.Gg, 75.30.Kz

Typeset Using REVTEX 


\section{INTRODUCTION}

We study the ground state properties of an $S=1 / 2$ alternating spin chain with the Hamiltonian

$$
H=\sum_{j}\left(S_{2 j}^{x} S_{2 j+1}^{x}+S_{2 j}^{y} S_{2 j+1}^{y}+\lambda S_{2 j}^{z} S_{2 j+1}^{z}\right)+\beta \sum_{j} \mathbf{S}_{2 j-1} \cdot \mathbf{S}_{2 j}
$$

where $\mathbf{S}_{j}=\left(S_{j}^{x}, S_{j}^{y}, S_{j}^{z}\right)$ are $S=1 / 2$ spin operators. This model has two exchange couplings

1 and $\beta$ alternately. When $\beta=1$ and $\lambda=1$, it is the spherically symmetric $S=1 / 2$ Heisenberg model which is solvable by the Bethe ansatz [1]. When $\beta=0$, the ground state is composed of local singlets that are formed by spins on sites $2 j$ and $2 j+1$. In the limit of strong ferromagnetic coupling $\beta \rightarrow-\infty$, spins on sites $2 j-1$ and $2 j$ form a local triplet. The first order degenerate perturbation in $1 / \beta$ from this limit gives the $S=1$ spin chain with the Hamiltonian

$$
H^{S=1}=\sum_{i}\left(S_{i}^{x} S_{i+1}^{x}+S_{i}^{y} S_{i+1}^{y}+\lambda S_{i}^{z} S_{i+1}^{z}\right)
$$

where $S_{i}^{x}, S_{i}^{y}$ and $S_{i}^{z}$ are $S=1$ spin operators at site $i$. When $\lambda=1$, the Hamiltonian (1.2) is the Heisenberg antiferromagnetic chain with $S=1$.

In 1983 Haldane conjectured that there are qualitative differences between integer and half-integer spin quantum antiferromagnetic chains based on the large- $S$ expansion [2]. While there is no excitation gap when $S$ is a half-integer [0], he argued that the Heisenberg Hamiltonian has a unique disordered ground state with a finite excitation gap when the spin $S$ is an integer. These claims have been confirmed by experimental [4, numerical [5 8] and analytical studies [9] at least for the $S=1$ case. The elementary excitations and the temperature dependence of the Haldane phase are investigated using the defect approach [10]. Although the ground state is disordered in the sense that the usual spin-spin correlation function decays exponentially, it has turned out that the ground state has a nontrivial hidden order. Den Nijs and Rommelse [11] argued that the Haldane phase is characterized by the hidden antiferromagnetic order based on the analogy of the preroughning transition. 
It is measured by the string order parameter. Kennedy and Tasaki [12] introduced a nonlocal unitary transformation which reveals the relation between the hidden antiferromagnetic order and the hidden discrete $Z_{2} \times Z_{2}$ symmetry breaking. The hidden $Z_{2} \times Z_{2}$ symmetry is completely broken in the Haldane phase. This is confirmed numerically [13]. Extensions to higher integer $S$ cases were discussed by several authors [14].

The $S=1 / 2$ spin chains with bond alternation have been investigated by several groups [15]. Recently Hida studied an $S=1 / 2$ Heisenberg chain with alternating ferromagnetic and antiferromagnetic couplings. Its Hamiltonian is (1.1) with $\lambda=1$. Hida considered a string order parameter for $S=1 / 2$ which leads to the den Nijs-Rommelse string order parameter in the $\beta \rightarrow-\infty$ limit. He concluded numerically that the gap and the string order parameter for $\beta=0$ remain non-vanishing in the $\beta \rightarrow-\infty$ limit. It suggests that the $S=1 / 2$ spin chains with disordered ground state are characterized by the string order parameters, and that for $\lambda=1$ there is no phase transition between $\beta=0(S=1 / 2$ local singlet-triplet gap) and $\beta=-\infty(S=1$ Haldane gap), thus they belongs to the same phase 16 18.

There are studies on the $S=1 / 2$ spin chains with bond alternation using other types of nonlocal unitary transformation [19,20]. In particular, Kohmoto and Tasaki [19] studied the Hamiltonian (1.1) by the nonlocal unitary transformation used in the work of the AshkinTeller model by Kohmoto, den Nijs and Kadanoff [21]. They found that this transformation plays a role of the Kennedy-Tasaki unitary transformation for the $S=1$ chain, although they are distinct. The nonlocal unitary transformation maps the $S=1 / 2$ alternating chain to an $S=1 / 2$ quantum spin system which is similar to the highly anisotropic version (onedimensional quantum system) of the two-dimensional Ashkin-Teller model. It also maps the string order parameters of the original chain to the ferromagnetic "local" order parameters in the transformed system. They argued the correspondence between the breaking of the $Z_{2} \times Z_{2}$ symmetry in the transformed system and the hidden antiferromagnetic order in the original system. In the transformed system, they showed that the region including the decoupled model $(\beta=0)$ and the $S=1$ chain are characterized by a full breakdown of the 
$Z_{2} \times Z_{2}$ symmetry. The Néel ordered phase (in terms of $S=1$ ) is characterized by a partial breakdown. They also proposed a ground state phase diagram.

The unitary transformation maps the quantum spin chain with bond alternation into the Ashkin-Teller type quantum spin system on a pair of chains. The spin chains are similar to the Ashkin-Teller quantum chain. The latter model is obtained by a highly anisotropic limit of the two-dimensional Ashkin-Teller model [21,22]. The two-dimensional Ashkin-Teller model [23] consists of two Ising models on a square lattice coupled by a four-spin interaction. In the time-continuum Hamiltonian formalism, a two-dimensional classical system is reduced to a one-dimensional quantum system by taking the extreme lattice anisotropic limit. The transfer-matrix method is used to convert a statistical mechanics problem at a finite temperature in two dimensions into a ground state problem for one-dimensional quantum Hamiltonian [24]. In this formalism, the ground state energy of a quantum system is the free energy of the corresponding two-dimensional classical system. The excitation gap in the quantum system corresponds to the inverse of the correlation length of the classical system. If there is no excitation gap in the quantum system, the classical system is critical. Namely phase boundaries of the ground states phase diagram of a one-dimensional quantum system correspond to critical points of the two-dimensional classical spin system. Thus we shall use the concepts of the critical phenomena (universality, critical indices etc.) in order to discuss the ground state phase diagram of the one-dimensional quantum system even in the parameter region where a corresponding two-dimensional classical system does not exist.

The purpose of the present paper is to determine the phase diagram of the Hamiltonian (1.1) quantitatively by a series expansion technique. Some limiting cases are considered analytically. The critical lines and critical indices are evaluated from the Padé method [25]. The obtained phase diagram shows a rich structure. It contains the gapful Haldane phase, the Néel phases, the $X Y$-like gapless phase. It also has a critical line with continuously varying critical indices. 


\section{THE DISORDER OPERATORS AND THE $Z_{2} \times Z_{2}$ SYMMETRY BREAKINGS}

We shall perform series expansions in terms of $\beta$. The unperturbed system $((\sqrt{1.1})$ with $\beta=0$ ) has the ground state which is a collection of uncorrelated local singlets. It is disordered in the sense that the expectation values of local order parameters vanish and series expansions cannot be applied to these quantities. Thus we consider the disorder operators which are nonzero in disordered phases and zero in an ordered phase [26]. We choose the following disorder operators

$$
D^{x y}(j)=\prod_{k>j}\left\{-2\left(S_{2 k}^{x} S_{2 k+1}^{x}+S_{2 k}^{y} S_{2 k+1}^{y}\right)\right\}=\prod_{k>j}\left\{-\left(S_{2 k}^{+} S_{2 k+1}^{-}+S_{2 k}^{-} S_{2 k+1}^{+}\right)\right\}
$$

and

$$
D^{z}(j)=\prod_{k>j}\left(-4 S_{2 k}^{z} S_{2 k+1}^{z}\right)
$$

to characterize the ground states of the Hamiltonian (1.1), where $S^{\alpha}$ are the $S=1 / 2$ spin operators.

We describe the derivation of above disorder operators and their relations to the hidden $Z_{2} \times Z_{2}$ symmetry. The nonlocal unitary transformation reveals the $Z_{2} \times Z_{2}$ symmetry of the Hamiltonian (1.1). This transformation is exactly the same as that used by Kohmoto, den Nijs and Kadanoff [21], which maps the staggered $X X Z$ model into the Ashkin-Teller quantum chain. Applying this transformation to the Hamiltonian (1.1), we get

$$
H=-\sum_{i}\left(\sigma_{i}^{x}+\tau_{i}^{x}+\lambda \sigma_{i}^{x} \tau_{i}^{x}\right)-\beta \sum_{i}\left(\sigma_{i}^{z} \sigma_{i+1}^{z}+\tau_{i}^{z} \tau_{i+1}^{z}+\sigma_{i}^{z} \sigma_{i+1}^{z} \tau_{i}^{z} \tau_{i+1}^{z}\right)
$$

where $\sigma_{i}^{\alpha}$ and $\tau_{i}^{\alpha}(\alpha=x, y$ and $z)$ are different kinds of Pauli matrices at site $i$. For details, see Appendix B of Ref. 21.

This Hamiltonian has symmetries of the dihedral group of order 4 which includes several $Z_{2}$ symmetries. There are many varieties of order parameters to measure the spontaneous symmetry breaking of these symmetries. We choose the following order operators:

$$
O_{ \pm}=\frac{\sigma_{i}^{z}+\tau_{i}^{z}}{2}
$$




$$
O_{2}=\sigma_{i}^{z} \tau_{i}^{z}
$$

The disorder operators which are dual to these order operators are

$$
\begin{gathered}
D_{ \pm}(i)=\prod_{k>i} \frac{\sigma_{k}^{x}+\tau_{k}^{x}}{2} \\
D_{2}(i)=\prod_{k>i} \sigma_{k}^{x} \tau_{k}^{x}
\end{gathered}
$$

respectively. These disorder operators have nonzero expectation values in disordered phases. Applying the inverse of the above transformation to the disorder operators (2.6) and (2.7), we get the disorder operators (2.1) and (2.2) of the Hamiltonian (1.1).

A nonzero expectation value of one of the disorder operators corresponds to a spontaneous breaking of a $Z_{2}$ symmetry. The ground state phases are characterized by a full breaking, partial breakings and nonbreakings of the $Z_{2} \times Z_{2}$ symmetry. Thus the ground state phase diagram can be obtained from analysis of the disorder operators (2.1) and (2.2).

The disorder operators (2.1) and (2.2) are related to the string order parameters of den Nijs and Rommelse, and Hida. It is expected that they have the same critical properties.

\section{PHASE DIAGRAM}

Before going to the detailed analysis, we summarize our results by showing the obtained phase diagram in Fig. 1. This phase diagram have seven phases and eight critical lines.

(1) The gapful Haldane phase (A). It is characterized by $\left\langle D^{\alpha}\right\rangle \neq 0,(\alpha=x y$ or $z)$.

(2) The $S=1$ Néel phase (B) which is characterized by $\left\langle D^{z}\right\rangle \neq 0$ and $\left\langle D^{x y}\right\rangle=0$. This region continuously connects to the Néel phase of the $S=1$ Hamiltonian (1.2).

(3) The $X Y$-like gapless phase (C) where $\left\langle D^{\alpha}\right\rangle(\alpha=x y$ and $z)$ vanishes. The correlation function decays algebraically in the entire region and the critical indices vary continuously.

(4) The ferromagnetic phase (D).

(5) The disordered phase (E) in which the ground state is a disordered dimer state with $\left\langle D^{\alpha}\right\rangle=0(\alpha=x y$ and $z)$. 
(6) The $S=1 / 2$ Néel ordered phase $(\mathrm{F})$. This phase is characterized by $\left\langle D^{z}\right\rangle \neq 0$ and $\left\langle D^{x y}\right\rangle=0$.

(7) The Néel ordered phase $(\mathrm{G})$ where spins on sites $2 j$ and $2 j+1$ prefer to align parallel and those on sites $2 j-1$ and $2 j$ prefer to align antiparallel.

These phases are separated by the following critical lines.

(1) Line 1 is expected to be in the Gaussian model universality class [27]. Critical indices vary continuously on this line for $-1<\lambda<1$. This critical line is expected to bifurcate at $\lambda=1[28]$.

(2) Line 2 and Line 3 are expected to be in the Ising model universality class. Line 2 approaches the line $\beta=2$ for large $\lambda$. Line 3 approaches the line $\beta=\frac{1}{2} \lambda$ for large $\lambda$.

(3) Line 4 is the phase boundary between the Haldane phase and the Néel phase (in terms of the $S=1$ model) and belongs to the Ising model universality class.

(4) Line 5 is the phase boundary between the $X Y$-like gapless phase and the Haldane phase, and is expected to be in the universality class of the Kosterlitz-Thouless transition [29].

(5) Line $6(\lambda=-1, \beta<0)$ is the phase boundary between the $X Y$-like phase and the ferromagnetic phase, and is expected to be in the universality class of the KDP transition. 30

(6) The Hamiltonian (1.1) is solvable on line $\beta=0$. The ground state is degenerate on Line 7. Thus, Line 7 is a critical line [21].

(7) Line 8 belongs to the Ising model universality class and approaches the line $\beta=-\frac{1}{2} \lambda$, for large $|\lambda|$.

\section{A. Limiting Cases}

In this subsection we discuss the phase diagram in several limits. Some of the critical lines are obtained by the duality. 


\section{1. limit $\lambda \gg|\beta| \sim 1$}

Since the term with $\lambda$ dominates, we can restrict ourselves to the space spanned by

$$
\left|\left\{\alpha_{j}\right\}\right\rangle=\bigotimes_{j}\left|\alpha_{j}\right\rangle_{2 j, 2 j+1}
$$

where the state $\left|\alpha_{j}\right\rangle_{2 j, 2 j+1}$ is defined by

$$
\left|\alpha_{j}=\uparrow\right\rangle_{2 j, 2 j+1}=|\uparrow\rangle_{2 j}|\downarrow\rangle_{2 j+1}, \quad\left|\alpha_{j}=\downarrow\right\rangle_{2 j, 2 j+1}=|\downarrow\rangle_{2 j}|\uparrow\rangle_{2 j+1} .
$$

Here, $|\uparrow\rangle_{j}$ and $|\downarrow\rangle_{j}$ are eigenstates of $S_{j}^{z}$ with eigenvalues $+1 / 2$ and $-1 / 2$ respectively. We treat the other parts of the Hamiltonian by the degenerate perturbation theory within the space spanned by (3.1). The first order perturbation gives the effective Hamiltonian

$$
H=\frac{1}{2} \sum_{i} \sigma_{i}^{x}-\frac{1}{4} \beta \sum_{i} \sigma_{i}^{z} \sigma_{i+1}^{z},
$$

where $\sigma_{i}^{\alpha}$ are the Pauli matrices which operate on $\left|\alpha_{i}\right\rangle_{2 i, 2 i+1}$. This is the highly anisotropic version of the two-dimensional Ising model [24]. This Hamiltonian have the property

$$
-\frac{\beta}{2} H\left(\frac{4}{\beta}, \widetilde{\sigma^{\alpha}}\right)=H\left(\beta, \sigma^{\alpha}\right),
$$

under the dual transformation

$$
\widetilde{\sigma_{i}^{z}}=\prod_{k=1}^{i} \sigma_{k}^{x}, \quad \widetilde{\sigma_{i}^{x}}=\sigma_{i}^{z} \sigma_{i+1}^{z} .
$$

For $\beta<0$, this duality of the Ising model tells us that $\beta=-2$ is the critical line. Thus the critical line between the Haldane phase and Néel phase approaches $\beta=-2$ as $\lambda$ becomes large. Above this line (phase A in Fig. 1), the $Z_{2} \times Z_{2}$ symmetry is fully broken. Below this line (phase B in Fig. 1), the ground state breaks half of the $Z_{2} \times Z_{2}$ symmetry. Critical properties corresponding to a partial breakdown of $Z_{2} \times Z_{2}$ symmetry is considered as the Ising model universality class. Thus Line 4 belongs to the Ising model universality class.

For $\beta>0$, we apply a transformation $S_{2 j}^{x} \rightarrow-S_{2 j}^{x}, S_{2 j}^{y} \rightarrow-S_{2 j}^{y}$ and $S_{2 j}^{z} \rightarrow S_{2 j}^{z}$ only for spins on the $2 j$ sites. Then we get the same duality relation as (3.4) except for the sign of $\beta$. It implies that $\beta=2$ is a self-dual line for the effective Hamiltonian. Thus Line 2 which approaches $\beta=2$ as $\lambda$ becomes large belongs to the Ising model universality class. 


\section{2. $\operatorname{limit} \beta, \lambda \gg 1$}

The Hamiltonian is approximated by

$$
H=\lambda \sum_{j} S_{2 j}^{z} S_{2 j+1}^{z}+\beta \sum_{j} \mathbf{S}_{2 j-1} \cdot \mathbf{S}_{2 j}
$$

When $\lambda$ is sufficiently large, the $\lambda$-coupling dominates in the Hamiltonian (3.6) and spins on sites $2 j$ and $2 j+1$ must form a state $|\uparrow\rangle_{2 j}|\downarrow\rangle_{2 j+1}$ or $|\downarrow\rangle_{2 j}|\uparrow\rangle_{2 j+1}$. In this bases, spins on sites $2 j-1$ and $2 j$ favors the states, $|\uparrow\rangle_{2 j-1}|\downarrow\rangle_{2 j}$ or $|\downarrow\rangle_{2 j-1}|\uparrow\rangle_{2 j}$, due to the antiferromagnetic coupling $\beta$. Therefore, the ground state is given either by $\bigotimes_{j}\left(|\uparrow\rangle_{2 j}|\downarrow\rangle_{2 j+1}\right)$ or $\bigotimes_{j}\left(|\downarrow\rangle_{2 j} \mid \uparrow\right.$ \rangle$\left._{2 j+1}\right)$. It is a doubly degenerate Néel ordered state. The energy per site is $\frac{1}{8} \lambda-\frac{1}{8} \beta$. On the other hand, spins on sites $2 j-1$ and $2 j$ favor to form singlet for $\beta \gg \lambda$. The ground state is given by an array of them. It is disordered and the energy is $-\frac{3}{8} \beta$.

To investigate the phase boundary between these phases, we map the Hamiltonian (3.6) into the model without bond alternation. Applying the nonlocal unitary transformation (see Sec. II) to the Hamiltonian (3.6), we get (see (2.3))

$$
H=-\lambda \sum_{i} \sigma_{i}^{x} \tau_{i}^{x}-\beta \sum_{i}\left(\sigma_{i}^{z} \sigma_{i+1}^{z}+\tau_{i}^{z} \tau_{i+1}^{z}+\sigma_{i}^{z} \sigma_{i+1}^{z} \tau_{i}^{z} \tau_{i+1}^{z}\right)
$$

We map the space spanned by the eigenstates of $\sigma_{i}^{z}$ and $\tau_{i}^{z}$ to that spanned by the eigenstates of $\sigma_{i}^{z} \tau_{i}^{z}$ and $\tau_{i}^{z}$. We define the operator $\widehat{\tau_{i}^{z}}$ by $\widehat{\tau_{i}^{z}}=\sigma_{i}^{z} \tau_{i}^{z}$. Using this notation, the Hamiltonian (3.7) becomes

$$
\begin{aligned}
H & =-\lambda \sum_{i} \tau_{i}^{x}-\beta \sum_{i}\left(\widehat{\tau_{i}^{z}} \widehat{\tau_{i+1}^{z}}+\tau_{i}^{z} \tau_{i+1}^{z}+\widehat{\tau_{i}^{z}} \tau_{i}^{z} \widehat{\tau_{i+1}^{z}} \tau_{i+1}^{z}\right) \\
& =-\lambda \sum_{i} \tau_{i}^{x}-\beta \sum_{i}\left\{\left(1+\tau_{i}^{z} \tau_{i+1}^{z}\right) \widehat{\tau_{i}^{z}} \widehat{\tau_{i+1}^{z}}+\tau_{i}^{z} \tau_{i+1}^{z}\right\} .
\end{aligned}
$$

In this Hamiltonian, the coupling of $\widehat{\tau_{i}^{z}}$ is negative, since $1+\tau_{i}^{z} \tau_{i+1}^{z}$ is always positive. Thus $\widehat{\tau}$ spins appear as the one-dimensional Ising model with the ferromagnetic coupling. In the ground state, $\widehat{\tau}$ spins are completely ordered ferromagnetically. We can replace the operator $\widehat{\tau_{i}^{z}} \widehat{\tau_{i+1}^{z}}$ by its expectation value $\left\langle\widehat{\tau_{i}^{z}} \widehat{\tau_{i+1}^{z}}\right\rangle=1$. Thus we have

$$
H=-\lambda \sum_{i} \tau_{i}^{x}-2 \beta \sum_{i} \tau_{i}^{z} \tau_{i+1}^{z}
$$


apart from a trivial additive constant. The effective Hamiltonian (3.10) is the Ising model in a transverse magnetic field. Therefore, the boundary of the phases is $\lambda=\frac{1}{2} \beta$ in this limit (Line 3 in Fig 1). Note that the phase transition for $\tau$ spins across critical line 3. Since the Hamiltonian (3.7) is symmetric with respect to $\tau$ spins and $\sigma$ spins, $\sigma$ spins have the same behavior as $\tau$ spins. Therefore, the order parameter (2.4) has nonzero expectation value in Phase E. The disorder operator whose series has singularity on Line 3 is $D^{z}$. Below this line (phase F in Fig 1), the system is ordered in terms of $S=1 / 2$ Néel order and is characterized by $\left\langle D^{z}\right\rangle \neq 0$ and $\left\langle D^{x y}\right\rangle=0$. Thus the ground state breaks half of the $Z_{2} \times Z_{2}$ symmetry. Above this line (phase E in Fig 1), the system is disordered and is characterized by $\left\langle D^{\alpha}\right\rangle=0(\alpha=x y$ and $z)$. The $Z_{2} \times Z_{2}$ symmetry is fully restored. Line 3 belongs to the Ising model universality class, since the $Z_{2}$ symmetry is broken across this line.

\section{3. $\operatorname{limit} \beta,|\lambda|(\lambda<0) \gg 1$}

Similar to the previous subsection, the Hamiltonian of this regime is

$$
H=-|\lambda| \sum_{j} S_{2 j}^{z} S_{2 j+1}^{z}+\beta \sum_{j} \mathbf{S}_{2 j-1} \cdot \mathbf{S}_{2 j}
$$

In the case of large $|\lambda|$, spins on sites $2 j$ and $2 j+1$ prefer to form a state $|\uparrow\rangle_{2 j}|\uparrow\rangle_{2 j+1}$ or $|\downarrow\rangle_{2 j}|\downarrow\rangle_{2 j+1}$. Spins on sites $2 j-1$ and $2 j$ favor the state $|\uparrow\rangle_{2 j}|\downarrow\rangle_{2 j+1}$ or $|\downarrow\rangle_{2 j} \mid \uparrow$ \rangle$_{2 j+1}$, since the coupling $\beta$ is antiferromagnetic. Therefore, the ground state is given by $\bigotimes_{j}\left(|\uparrow\rangle_{4 j}|\uparrow\rangle_{4 j+1}|\downarrow\rangle_{4 j+2}|\downarrow\rangle_{4 j+3}\right)$ and $\bigotimes_{j}\left(|\downarrow\rangle_{4 j}|\downarrow\rangle_{4 j+1}|\uparrow\rangle_{4 j+2}|\uparrow\rangle_{4 j+3}\right)$. The energy of this state is $-\frac{1}{8}|\lambda|-\frac{1}{8} \beta$ per site. For $\beta \gg|\lambda|$, spins on sites $2 j-1$ and $2 j$ favor a singlet state. The ground state is given by a collection of local singlet with the energy $-\frac{3}{8} \beta$. In this case, the phase boundary is the line $\lambda=-\frac{1}{2} \beta$ (Line 8 in Fig. 目). Below this line (Phase G in Fig. 1), the system breaks half of the $Z_{2} \times Z_{2}$ symmetry. Thus the transition across this line belongs to the Ising model universality class. 


$$
\text { 4. line } \beta=0
$$

We have the simplest situation. The Hamiltonian is written

$$
H=\sum_{j}\left(S_{2 j}^{x} S_{2 j+1}^{x}+S_{2 j}^{y} S_{2 j+1}^{y}+\lambda S_{2 j}^{z} S_{2 j+1}^{z}\right)
$$

It is a collection of independent two-spin systems. For $\lambda>-1$, the ground state is given by $\otimes_{j}\left\{\frac{1}{\sqrt{2}}\left(|\uparrow\rangle_{2 j}|\downarrow\rangle_{2 j+1}-|\downarrow\rangle_{2 j}|\uparrow\rangle_{2 j+1}\right)\right\}$ with an energy $-\frac{2+\lambda}{8}$ per site. For $\lambda<-1$, the ground state of an independent two-spin system is given by $|\uparrow\rangle_{2 j}|\uparrow\rangle_{2 j+1}$ or $|\downarrow\rangle_{2 j}|\downarrow\rangle_{2 j+1}$. Therefore, the ground state of the total system is $2^{M / 2}$ fold degenerate, where $M$ is the number of sites. Therefore, this is a critical line [21]. The energy is $\frac{\lambda}{8}$ per site.

\section{5. the ferromagnetic phase $\beta<0, \lambda<-1$}

In this region, the Hamiltonian is written

$$
H=\sum_{j}\left(S_{2 j}^{x} S_{2 j+1}^{x}+S_{2 j}^{y} S_{2 j+1}^{y}-|\lambda| S_{2 j}^{z} S_{2 j+1}^{z}\right)-|\beta| \sum_{j} \mathbf{S}_{2 j-1} \cdot \mathbf{S}_{2 j}
$$

The ground state is given by $\bigotimes_{j}|\uparrow\rangle_{2 j}|\uparrow\rangle_{2 j+1}$ or $\bigotimes_{j}|\downarrow\rangle_{2 j}|\downarrow\rangle_{2 j+1}$ with the energy $-\frac{|\lambda|+|\beta|}{8}$.

Line 6 is expected to be a critical line in the universality class of the KDP transition [30], since the system is in the perfectly ferromagnetic ordered state in the $\lambda<-1$ side and is in the $X Y$-like gapless phase in the $\lambda>-1$ side.

\section{6. limit $\beta \rightarrow-\infty$}

In the $\beta \rightarrow-\infty$ limit, the $S=1 / 2$ Hamiltonian (1.1) is equivalent to the $S=1 X X Z$ Hamiltonian (1.2). It has the Néel, the Haldane, the $X Y$-like and the ferromagnetic phases depending on the parameter $\lambda$. We denote $\lambda_{1}$ for the boundary between the Néel and the Haldane phases, $\lambda_{2}$ for that between the Haldane and the $X Y$-like phases, and $\lambda_{3}$ for the $X Y$-like and the ferromagnetic phases.

The phase boundary between the Néel and the Haldane phases is estimated to be $\lambda_{1} \sim 1.2$ numerically [5,7]. The critical line line 4 is expected to approach $\lambda=\lambda_{1}$ [19]. The boundary 
between the Haldane and the $X Y$-like phase is estimated $\lambda_{2} \sim 0$ numerically [5,8]. The $X Y$-like phase boundary which starts from the point $(\lambda, \beta)=(-1,0)$ approaches $\lambda=\lambda_{2}$.

For $\lambda=-1$, the $S=1 X X Z$ Hamiltonian (1.2) can be mapped to the ferromagnetic Heisenberg model by the rotation in the spin space for the $2 j$ sites, $S_{2 j}^{x} \rightarrow-S_{2 j}^{x}, S_{2 j}^{y} \rightarrow-S_{2 j}^{y}$

and $S_{2 j}^{z} \rightarrow S_{2 j}^{z}$. The ground state have degeneracy due to the rotational symmetry at $\lambda=-1$. On the other hand, for $\lambda<-1$, the ground state is 2 fold degenerate. Therefore, the phase boundary between the $X Y$-like and the ferromagnetic phases is $\lambda=-1$ in this limit.

\section{SERIES EXPANSION}

We will make a series expansion in terms of $\beta$ and obtain series of the "specific heat", the "magnetization" and the "susceptibility" to estimate critical points and critical indices.

\section{A. Outline of the Method}

It is necessary to have long series to extract reliable estimates of the critical points and critical indices. We use the linked cluster expansion method proposed by Kadanoff and Kohmoto [31]. It is suited to calculate high order terms effectively.

Hamiltonian (1.1) is written

$$
H=T+\beta V
$$

where

$$
T=\sum_{j}\left(S_{2 j}^{x} S_{2 j+1}^{x}+S_{2 j}^{y} S_{2 j+1}^{y}+\lambda S_{2 j}^{z} S_{2 j+1}^{z}\right)
$$

and

$$
V=\sum_{j} \mathbf{S}_{2 j-1} \cdot \mathbf{S}_{2 j}
$$

We shall perform series expansions with respect to $\beta$. The unperturbed states are 


$$
\left|\left\{\alpha_{j}\right\}\right\rangle=\bigotimes_{j}\left|\alpha_{j}\right\rangle_{2 j, 2 j+1}
$$

where $\left|\alpha_{j}\right\rangle_{2 j, 2 j+1}$ is one of four eigenstates of the operator $S_{2 j}^{x} S_{2 j+1}^{x}+S_{2 j}^{y} S_{2 j+1}^{y}+\lambda S_{2 j}^{z} S_{2 j+1}^{z}$ : $|S\rangle_{2 j, 2 j+1}=\frac{1}{\sqrt{2}}\left(|\uparrow\rangle_{2 j}|\downarrow\rangle_{2 j+1}-|\downarrow\rangle_{2 j}|\uparrow\rangle_{2 j+1}\right),\left|T_{+1}\right\rangle_{2 j, 2 j+1}=|\uparrow\rangle_{2 j}|\uparrow\rangle_{2 j+1},\left|T_{0}\right\rangle_{2 j, 2 j+1}=$ $\frac{1}{\sqrt{2}}\left(|\uparrow\rangle_{2 j}|\downarrow\rangle_{2 j+1}+|\downarrow\rangle_{2 j}|\uparrow\rangle_{2 j+1}\right)$ and $\left|T_{-1}\right\rangle_{2 j, 2 j+1}=|\downarrow\rangle_{2 j}|\downarrow\rangle_{2 j+1}$. For $\lambda>-1$, the unperturbed ground state is

$$
|G\rangle=\bigotimes_{j}|S\rangle_{2 j, 2 j+1}
$$

The ground state energy is expanded in a power series as

$$
E(\lambda, \beta)=\sum_{n=0} E^{(n)}(\lambda) \beta^{n} .
$$

The "specific heat" is obtained by

$$
C(\lambda, \beta)=\frac{\partial^{2}}{\partial \beta^{2}} E(\lambda, \beta) .
$$

We use the disorder operators defined per site

$$
\mathcal{D}^{\alpha}=\frac{1}{M} \sum_{j} D^{\alpha}(j)
$$

where $\alpha=x y$ and $z$, and $M$ is the number of sites. To calculate series for the "magnetization" of the operators, we simply add a magnetic field

$$
H=T+\beta V+h \mathcal{D}^{\alpha} .
$$

The ground state energy in the presence of $h$ is calculated in a power series of $\beta$ and $h$ as

$$
E(\lambda ; \beta, h)=\sum_{n=0} \sum_{m=0} E^{(n, m)}(\lambda) \beta^{n} h^{m} .
$$

The expectation value of the "magnetization" is given by

$$
\left\langle\mathcal{D}^{\alpha}\right\rangle=\left.\frac{\partial E(\lambda ; \beta, h)}{\partial h}\right|_{h=0},
$$

and the "susceptibility" is 


$$
\frac{\partial\left\langle\mathcal{D}^{\alpha}\right\rangle}{\partial h}=\left.\frac{\partial^{2} E(\lambda ; \beta, h)}{\partial h^{2}}\right|_{h=0} .
$$

We use the Padé method to estimate critical points $\beta_{c}$ and critical indices. The definition of critical indices are as follows:

$$
\begin{gathered}
\frac{\partial^{2}}{\partial \beta^{2}} E(\lambda, \beta) \sim \text { constant }\left|\frac{\beta_{c}-\beta}{\beta_{c}}\right|^{-\alpha}, \quad\left(\beta \rightarrow \beta_{c}\right), \\
\left.\frac{\partial E(\lambda ; \beta, h)}{\partial h}\right|_{h=0} \sim \text { constant }\left|\frac{\beta_{c}-\beta}{\beta_{c}}\right|^{-\beta_{j}}, \quad\left(\beta \rightarrow \beta_{c}\right), \\
\left.\frac{\partial^{2} E(\lambda ; \beta, h)}{\partial h^{2}}\right|_{h=0} \sim \text { constant }\left|\frac{\beta_{c}-\beta}{\beta_{c}}\right|^{-\gamma_{j}}, \quad\left(\beta \rightarrow \beta_{c}\right),
\end{gathered}
$$

where $j$ is $x y$ and $z$.

\section{B. Result from Series Analysis}

The quantities calculated by series expansions were (1) the "specific heat" (13 terms), (2) the "magnetization" (15 terms), (3) the "susceptibility" (13 terms). Critical points and critical indices are evaluated by the Dlog Padé method [32]. The estimates for the quantities are obtained by averaging the three or four highest-order diagonal elements and near-diagonal elements of the Padé tables. Error bars are then set to include these three or four values.

\section{1. region $\beta>0$}

For the region $-1<\lambda<1$, the Haldane phase is characterized by $\left\langle\mathcal{D}^{\alpha}\right\rangle \neq 0(\alpha=x y$ and $z)$. The disordered phase $(\mathrm{E})$ is characterized by $\left\langle\mathcal{D}^{\alpha}\right\rangle=0(\alpha=x y$ and $z)$. Thus the phase boundary (Line 1) between these phases is determined from series for $\mathcal{D}^{\alpha}(\alpha=x y$ and $z$ ). Critical points obtained from series for $\left\langle\mathcal{D}^{x y}\right\rangle$ and $\left\langle\mathcal{D}^{z}\right\rangle$ show well convergence. We estimate critical points from series for $\left\langle\mathcal{D}^{x y}\right\rangle$ (Fig. 1). In Fig. 目 and 3, critical indices $\beta_{j}$ 's $(j$ 
$=x y$ and $z)$ are shown. Results for $\gamma_{j}{ }^{\prime}$ s $(j=x y$ and $z)$ and $\alpha$ are shown in Fig. 囵, 5 and 6 respectively. In this region, critical indices $\beta_{j}$ 's $(j=x y$ and $z)$ vary continuously, and seem to be divergent near $\lambda=-1$. Critical index $\beta_{z}$ agrees well with the result by Hida obtained numerically [33]. Critical indices $\gamma_{j}$ 's $(j=x y$ and $z)$ and $\alpha$ are also varying continuously. Critical index $\gamma_{x y}$ also seems to be divergent near $\lambda=-1$. However, we cannot determine critical indices accurately close to $\lambda=-1$ due to poor convergence. In the case of the Ashkin-Teller quantum chain or the staggered $X X Z$ model, there is the "critical fan" in a finite region of the parameter space $-1<\lambda<-\frac{1}{\sqrt{2}}$ [21]. The "critical fan" is a region where a line of continuously varying criticality "fans out" and becomes an area of critical behavior. In that case, critical indices show divergence near $\lambda=-\frac{1}{\sqrt{2}}$ on the self-dual line of the Ashkin-Teller quantum chain. In the present model, there is no sign of a "critical fan" within this analysis, since critical points show well convergence and critical indices does not show divergence. However, we cannot discuss the existence of the "critical fan" near $\lambda=-1$ for poor convergence of critical indices.

For $\beta=1, \lambda=1$, the model is solvable by the Bethe ansatz [1]. We can map it to the six-vertex model at this point [34. The extended scaling relations predict critical indices $\alpha, \beta_{j}$ 's and $\gamma_{j}$ 's $(j=x y$ and $z)$, with the help of the standard scaling relations. These indices have been obtained from mappings between the six-vertex model and the Gaussian model [27,35]. The equivalent mapping is that between the $X X Z$ model and the TomonagaLuttinger model [36]. The estimated values of the critical point from the series analysis are, $\beta_{c}=1.003 \pm 0.002$ (from series for the "specific heat"), $\beta_{c}=1.00168 \pm 0.00004$ (from series for $\left.\left\langle\mathcal{D}^{x y}\right\rangle\right), \beta_{c}=0.99782 \pm 0.00009$ (from series for $\left\langle\mathcal{D}^{x y} \mathcal{D}^{x y}\right\rangle$ ). The critical indices $\alpha, \beta_{j}$ 's and $\gamma_{j}^{\prime}$ 's $(j=x y$ and $z)$ are

$$
\begin{gathered}
\alpha=0.55 \pm 0.01 \quad\left(\frac{2}{3}=0.666 \cdots\right), \\
\beta_{j}=0.08202 \pm 0.00008 \quad\left(\frac{1}{12}=0.0833 \cdots\right), \\
\gamma_{j}=1.23 \pm 0.02 \quad\left(\frac{7}{6}=1.166 \cdots\right) .
\end{gathered}
$$


The values in parentheses are the expected values from the mappings.

Next we consider the region $\lambda>1$. The Haldane phase is characterized by $\left\langle\mathcal{D}^{\alpha}\right\rangle \neq 0$ $\left(\alpha=x y\right.$ and $z$ ). The $S=1 / 2$ Néel phase $(\mathrm{F})$ is characterized by $\left\langle\mathcal{D}^{x y}\right\rangle=0$ and $\left\langle\mathcal{D}^{z}\right\rangle \neq 0$. Thus the phase boundary (Line 2) between these phases is determined from series for $\mathcal{D}^{x y}$. In Phase E, both of $\left\langle\mathcal{D}^{x y}\right\rangle$ and $\left\langle\mathcal{D}^{z}\right\rangle$ vanish. Therefore, the phase boundary (Line 3) is determined from series for $\mathcal{D}^{z}$. These results are shown in Fig. 1. Line 2 and Line 3 belong to the Ising model universality class (see Sec. [II]). Thus critical indices $\alpha, \beta_{x y}$ and $\gamma_{x y}$ must be $0, \frac{1}{8}$ and $\frac{7}{4}$, respectively on Line 2 . Critical indices $\alpha, \beta_{z}$ and $\gamma_{z}$ must be $0, \frac{1}{8}$ and $\frac{7}{4}$, respectively on Line 3 . The series analysis shows that Line 2 approaches the line $\beta_{c}=2$, which is consistent with the result in Sec. III. For $\lambda>1$, Critical index $\beta_{x y}$ agrees well with that of the Ising model $\frac{1}{8}$. Critical index $\gamma_{x y}$ also agrees well with that of the Ising model $\frac{7}{4}$. Near $\lambda=1$ they do not agree with the Ising values. We regard this as a numerical effect. Critical index $\alpha$ does not agree well with the Ising value $\alpha=0$ as $\lambda$ becomes large. In the series analysis for $\left\langle\mathcal{D}^{z}\right\rangle$, there is a pole near Line 2. We choose the second pole to determine the critical points and critical indices for Line 3 as shown in Fig. 1. The operator $\mathcal{D}^{z}$ has nonzero expectation value in Phase $\mathrm{A}$ and in Phase $\mathrm{F}$. We regard the first pole as unphysical consequences, since series for $\mathcal{D}^{z}$ has no singularity on Line 2 . The series analysis shows that Line 3 approaches the line $\beta=\frac{1}{2} \lambda$. This is consistent with the result in Sec. [II. However, their convergence is poor. Critical indices $\alpha, \beta_{z}$ and $\gamma_{z}$ are not included here for poor convergence. Critical indices $\beta_{z}$ estimated from series analysis are $\beta_{z}=0.03 \sim 0.1$ for $\lambda=2 \sim 5$. They do not agree well with the Ising values. Critical index $\gamma_{z}$ does not converge. We think that these are due to the first pole near Line 2 and large value of $\beta_{c}$.

\section{2. region $\beta<0$}

Let us consider the region $\lambda>0$. The $S=1$ Néel phase (B) is characterized by $\left\langle\mathcal{D}^{x y}\right\rangle=0$ and $\left\langle\mathcal{D}^{z}\right\rangle \neq 0$. Thus the phase boundary (Line 4) between the Haldane and the $S=1$ Néel phases is determined from series for $\mathcal{D}^{x y}$. The best estimates are obtained from series for 
$\left\langle\mathcal{D}^{x y}\right\rangle$ as shown in Fig. 1. Line 4 belongs to the Ising model universality class (see Sec. [II). Therefore, critical indices are expected to take the Ising values throughout Line 4 . In the limit $\beta \rightarrow-\infty$, Line 4 is expected to approach the Néel-Haldane transition point $\lambda_{1}$ of the $S=1 X X Z$ model. The critical points and critical indices obtained from series for $\left\langle\mathcal{D}^{x y}\right\rangle$ are well convergent for $\lambda \gtrsim 2.5$, and stable for $2 \lesssim \lambda \lesssim 2.5$. However, they are unstable for $\lambda \lesssim 2$. No pole was found in every highest-order element of Padé tables for $0<\lambda \lesssim 1.2$ except for them with small residue $\left(<10^{-5}\right)$. Note that if we investigate the behavior of Padé tables carefully, they show a highly unstableness near $\lambda \sim 1.8$. Thus, for the region $1.2 \lesssim \lambda \lesssim 1.8$, we regard the critical points as poor convergence of the Padé approximation. For large $\lambda$, the critical line approaches the line $\beta_{c}=-2$, which is consistent with the result in Sec. III]. Critical index $\beta_{x y}$ is well convergent to that of the Ising model $\frac{1}{8}$ (see Fig. 7). The result for $\gamma_{x y}$ is shown in Fig. 8. It shows a good agreement with the Ising value $\frac{7}{4}$. Convergence of critical indices become poor around $\lambda=2$. This is due to large value of $\beta_{c}$.

Next we focus on the region $-1<\lambda<0$. In the $X Y$-like phase, both of $\left\langle\mathcal{D}^{x y}\right\rangle$ and $\left\langle\mathcal{D}^{z}\right\rangle$ vanish. Thus the phase boundary (Line 5) between the Haldane and the $X Y$-like phases is determined from series for $\mathcal{D}^{\alpha}(\alpha=x y$ and $z)$. We choose the operator $\mathcal{D}^{z}$ to determine Line 5 , since critical points obtained from it converges better. The critical points and indices are stable near the point $(\lambda, \beta)=(-1,0)$. The convergence of them becomes poor when $\lambda$ approaches 0 , and it is difficult to determine the critical points and critical indices. Continuously varying critical exponents are observed for $\beta_{x y}$ and $\beta_{z}$ along this line, which are shown in Fig. 7 and Fig. 9 respectively. Critical indices $\alpha$ and $\gamma_{j}$ 's $(j=x y$ and z) are not included here for poor convergence. These values estimated from series analysis are $\alpha=2.2 \pm 1.1, \gamma_{x y}=3.4 \pm 2.6$ and $\gamma_{z}=3.1 \pm 2.6$ at $\lambda=-0.8$. Line 5 is expected to be in the universality class of the Kosterlitz-Thouless transition. However, we cannot evaluate critical index $\eta$ for poor convergence of critical indices $\gamma_{j}$ 's $(j=x y$ and $z)$. 


\section{SUMMARY AND DISCUSSION}

We studied the ground state properties of the $S=1 / 2$ quantum spin chain with bond alternation. This model is equivalent to the $S=1$ antiferromagnetic $X X Z$ model in the strong ferromagnetic coupling limit. We focused on the disorder operators. These operators are also obtained from the disorder operators of the Ashkin-Teller quantum chain by using the nonlocal unitary transformation. The "magnetization" of these nonlocal operators measure similar quantity that is observed by the string order parameters which is extended by Hida for the $S=1 / 2$ quantum spin chains.

The ground state phase diagram is obtained by series expansions. Critical points are consistent with the phase diagram which conjectured by Kohmoto and Tasaki [19] and determined numerically by Hida $[18$. In the narrow region $\lambda>-1, \beta \sim 0$, it can be proved rigorously that the ground state in the infinite volume limit is unique, all the truncated correlation functions decay exponentially, and there is a finite excitation gap [12,19]. The present study strongly suggest that this region covers the wide area surrounding by Lines 1 , 2, 4 and 5 in Fig. 四. The results of critical indices show that phase transition between the Haldane phase and the Néel phase (Line 4 in Fig. 1) belongs to the Ising model universality class. The Ising-like critical properties are originated from the partial breakdown of the $Z_{2} \times Z_{2}$ symmetry in this case. This supports that the hidden $Z_{2} \times Z_{2}$ symmetry breaking is to be a criterion to distinguish the Haldane gap system. Although the nonlocal unitary transformation discussed here is not exactly the same that used by Kohmoto and Tasaki, the disorder operators have same critical properties with theirs. This result also supports the proposal by Hida that the Haldane phase which is characterized by the string order parameter belongs to the same phase as that of the decoupled $S=1 / 2$ chain. 


\section{ACKNOWLEDGMENTS}

We wish to thank Kazuo Hida for useful discussions and for sending his results prior to publication. 


\section{REFERENCES}

* $\quad$ Staying at M.I.T. until Sep. 301993.

[1] H. A. Bethe, Z. Phys. 71, 205 (1931).

[2] F. D. M. Haldane, Phys. Lett. 93A, 464 (1983); Phys. Rev. Lett. 50, 1153 (1983).

[3] P. W. Anderson, Phys. Rev. 86, 694 (1952); R. Kubo, Phys. Rev. 87, 568 (1952); J. des Cloizeaux and J. J. Pearson, Phys. Rev. 128, 2131 (1962).

[4] W. J. L. Buyers, R. M. Morra, R. L. Armstrong, M. J. Hogan, P. Gerlack and K. Hirakawa, Phys. Rev. Lett. 56, 371 (1986); M. Steiner, K. Kakurai, J. K. Kjems, D. Petitgrand and R. Pynn, J. Appl. Phys. 61, 3953 (1987); J. P. Renard, M. Verdaguer, L. P. Regnault, W. A. C. Erkelens, J. Rossat-Mignod, J. Ribas, W. G. Stirling and C. Vettier, J. Appl. Phys. 63, 3583 (1988); K. Katsumata, H. Hori, T. Takeuchi, M. Date and A. Yamagishi and J. P. Renard, Phys. Rev. Lett. 63, 86 (1989); Y. Ajiro, T. Goto, H. Kikuchi, T. Sakakibara and T. Inami, Phys. Rev. Lett. 63, 1424 (1989); M. Hagiwara, K. Katsumata, I. Affleck, B. I. Halperin and J. P. Renard, Phys. Rev. Lett. 65, 3181 (1990).

[5] R. Botet, R. Jullien and M. Kolb, Phys. Rev. B28, 3914 (1983); H. J. Schulz and T. Ziman, Phys. Rev. B33, 6545 (1986).

[6] M. P. Nightingale and H. W. Blöte, Phys. Rev. B33, 659 (1986); M. Takahashi, Phys. Rev. Lett. 62, 2313 (1989); T. Sakai and M. Takahashi, Phys. Rev. B42, 1090 (1990).

[7] K. Nomura, Phys. Rev. B40, 9142 (1989).

[8] K. Kubo and S. Takada, J. Phys. Soc. Jpn, 55, 438 (1986); F. C. Alcaraz and Y. Hatsugai, Phys. Rev. B46, 13914 (1992).

[9] I. Affleck, T. Kennedy, E. H. Lieb and H. Tasaki, Phys. Rev. Lett. 59, 799 (1987); Commun. Math. Phys. 115, 477 (1988). 
[10] G. Gómez-Santos, Phys. Rev. Lett. 63, 790 (1989); H. -J. Mikeska, Europhys. Lett. 19, 39 (1992); H. Köhler and R. Schilling, J. Phys. Cond. Matter, 4, 7899 (1992).

[11] M. den Nijs and K. Rommelse, Phys. Rev. B40, 4709 (1989).

[12] T. Kennedy and H. Tasaki, Phys. Rev. B45, 304 (1992); H. Tasaki, Phys. Rev. Lett. 66, 798 (1991).

[13] S. M. Girvin and D. P. Arovas, Phys. Scr. T27, 156 (1989); Y. Hatsugai and M. Kohmoto, Phys. Rev. B44, 11789 (1991).

[14] H. Tasaki (private communication); M. Oshikawa, J. Phys. Cond. Matter, 4, 7469 (1992); Y. Hatsugai, J. Phys. Soc. Jpn, 61, 3856 (1992).

[15] T. Nakano and H. Fukuyama, J. Phys. Soc. Jpn. 49, 1679 (1980); J. Phys. Soc. Jpn. 50, 2489 (1981); K. Okamoto, H. Nishimori and Y. Taguchi, J. Phys. Soc. Jpn. 55, 1458 (1986); S. Yoshida and K. Okamoto, J. Phys. Soc. Jpn. 58, 4367 (1989); and references there in.

[16] K. Hida, Phys. Rev. B45, 2207 (1992).

[17] K. Hida, Phys. Rev. B46, 8268 (1992).

[18] K. Hida, Comp. Phys. as a New Frontier in Cond. Matt. Res. Proc. July 86 (1992); (and private communication).

[19] M. Kohmoto and H. Tasaki, Phys. Rev. B46, 3486 (1992)

[20] S. Takada and H. Watanabe, J. Phys. Soc. Jpn. 61, 39 (1992); S. Takada, J. Phys. Soc. Jpn. 61, 428 (1992).

[21] M. Kohmoto, M. den Nijs and L. P. Kadanoff, Phys. Rev. B24, 5229 (1981).

[22] L. P. Kadanoff and M. Kohmoto, Nucl. Phys. B190[FS3], 671 (1981).

[23] J. Ashkin and E. Teller, Phys. Rev. 64, 178 (1943). 
[24] For a review, see J. B. Kogut, Rev. Mod. Phys. 51, 659 (1979).

[25] For an example see D. S. Gaunt and A. J. Guttmann, in Phase Transitions and Critical Phenomena, edited by C. Domb and M. S. Green (Academic, London, 1974), Vol. 3.

[26] L. P. Kadanoff and H. Ceva, Phys. Rev. B3, 3918 (1971).

[27] L. P. Kadanoff and A. C. Brown, Ann. Phys. 121, 318 (1979).

[28] L. P. Kadanoff, Phys. Rev. B22, 1405 (1980).

[29] J. M. Kosterlitz and D. J. Thouless, J. Phys. C 6, 1181 (1973); J. M. Kosterlitz, J. Phys. C 7, 1046 (1974).

[30] E. H. Lieb, Phys. Rev. Lett. 18, 692, 1046 (1967); Phys. Rev. Lett. 19, 108 (1967); For a review see, E. H. Lieb and F. Y. Wu, in Phase Transitions and Critical Phenomena, edited by C. Domb and M. S. Green (Academic, London, 1972), Vol. 1.

[31] L. P. Kadanoff and M. Kohmoto, J. Phys. A 14, 1291 (1981).

[32] G. A. Baker Jr., Phys. Rev. 124, 768 (1961).

[33] K. Hida (unpublished and private communication).

[34] R. J. Baxter, Phys. Rev. Lett. 26, 832 (1971); M. Barbar and R. J. Baxter, J. Phys. C 6, 2913 (1973); L. P. Kadanoff, Phys. Rev. Lett. 39, 903 (1977).

[35] L. P. Kadanoff, Ann. Phys. (N.Y.) 120, 39 (1979); H. J. F. Knops, Ann. Phys. (N.Y.) 128, 448 (1981).

[36] A. Luther and I. Peschel, Phys. Rev. B12, 3908 (1975); M. den Nijs, Phys. Rev. B23, 6111 (1981); J. L. Black and V. J. Emery, Phys. Rev. B23, 429 (1981). 


\section{FIGURES}

FIG. 1. Phase diagram of the alternating quantum spin chain with the Hamiltonian (1.1). Estimates of critical points by a series analysis are shown with error bars. For those without an error bar, the error is smaller than the size of the plotted point.

FIG. 2. Critical index $\beta_{x y}$ as a function of $\lambda$ on Line 1 and 2 .

FIG. 3. Critical index $\beta_{z}$ as a function of $\lambda$ on Line 1 .

FIG. 4. Critical index $\gamma_{x y}$ as a function of $\lambda$ on Line 1 and 2.

FIG. 5. Critical index $\gamma_{z}$ as a function of $\lambda$ on Line 1.

FIG. 6. Critical index $\alpha$ as a function of $\lambda$ on Line 1 and 2.

FIG. 7. Critical index $\beta_{x y}$ as a function of $\lambda$ on Line 4 and 5 .

FIG. 8. Critical index $\gamma_{x y}$ as a function of $\lambda$ on Line 4 .

FIG. 9. Critical index $\beta_{z}$ as a function of $\lambda$ on Line 5 . 